This is the post peer-review accepted manuscript of:

F. Guidi, N. Decarli, D. Dardari, F. Mani and R. D'Errico, "Passive Millimeter-Wave RFID Using Backscattered Signals," 2016 IEEE Globecom Workshops (GC Wkshps), Washington, DC, 2016, pp. 1-6.

https://doi.org/10.1109/GLOCOMW.2016.7848994

The published version is available online at:

http://ieeexplore.ieee.org/stamp/stamp.jsp?tp=\&arnumber=7848994\&isnumber=7848793

C 2016 IEEE. Personal use of this material is permitted. Permission from IEEE must be obtained for all other uses, in any current or future media, including reprinting/republishing this material for advertising or promotional purposes, creating new collective works, for resale or redistribution to servers or lists, or reuse of any copyrighted component of this work in other works 


\title{
Passive Millimeter-Wave RFID using Backscattered Signals
}

\author{
Francesco Guidi ${ }^{\dagger \ddagger}$, Nicoló Decarli*, Davide Dardari*, Francesco Mani ${ }^{\dagger \ddagger}$ and Raffaele D’Errico ${ }^{\dagger \ddagger}$ \\ ${ }^{\dagger}$ CEA, LETI, MINATEC Campus, 38054 Grenoble, France \\ $\ddagger$ Univ. Grenoble-Alpes, 38000 Grenoble, France \\ \{francesco.guidi, francesco.mani, raffaele.derrico\}@cea.fr \\ *DEI, University of Bologna, via Venezia 52, I-47521 Cesena (FC), Italy \\ \{nicolo.decarli, davide.dardari\}@unibo.it
}

\begin{abstract}
In this paper we investigate the possibility to jointly adopt millimeter-wave technology with passive RFID based on backscattered signals, which paves the way to new identification and localization applications for next $5 \mathrm{G}$ scenarios. In particular, we assess the feasibility of this solution by comparing it with the passive RFID in the microwave UWB band. Results show that, despite the high path-loss at $60 \mathrm{GHz}$, it is possible to achieve interesting performance thanks to the less stringent requirements in terms of emitted power with respect to the UWB technology. Moreover, the possibility of localizing tags with a single reader is foreseen thanks to the adoption of narrow-beam antennas and beamsteering operations.
\end{abstract}

Index Terms-RFID, Millimeter-Waves, Backscatter Modulation

\section{INTRODUCTION}

Next Internet of Things (IoT) and fifth generation (5G) communication scenarios are expected to bring an increased connectivity between people and the surrounding environment, foreseeing a pervasive presence of tagged objects [1]. Among the expected key technologies, radiofrequency identification (RFID) has attracted a great interest in the recent years. It has been adopted to overcome current bar-codes limitations in the short reading distance, with the opportunity to detect and identify batteryless tags attached to objects placed even at several meters from the reader. Current Gen. 2 ultra-high frequency (UHF)-RFID is widely exploited and studied [2], and new solutions have been proposed in order to enable tags localization [3]. Recent studies have moved from UHF band to the ultrawide bandwidth (UWB) technology in the $3.1-10.6 \mathrm{GHz}$ microwave bandwidth in order to integrate the possibility to localize tags as well as to reduce the power consumption [4], [5]. In particular, the use of passive UWBRFID solutions has a great appeal in all those applications requiring low-cost as well as good ranging performance. Since passive tags are not equipped with a transmitter, the communication relies on the modulation of the backscattered signal and several techniques were conceived in order to maximize the performance [6], [7]. Unfortunately, due to power regulations constraints, it has been shown that passive UWB is strongly limited in terms of reader-tag communication range. Moreover, high accuracy localization requires multiple interrogations from at least three readers.

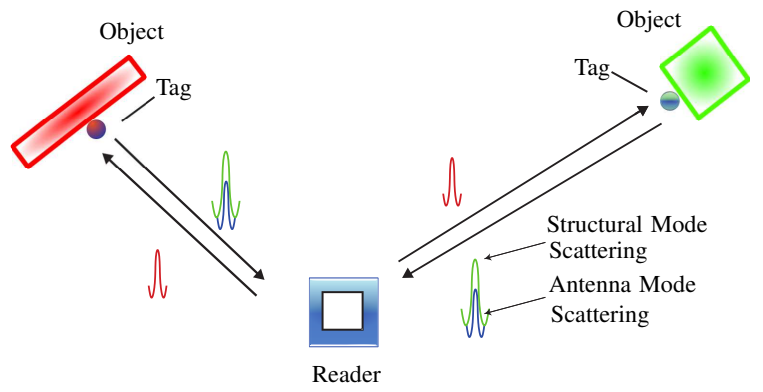

Fig. 1. Considered RFID scenario.

To overcome such limitation, the joint adoption of RFID and millimeter-wave $(\mathrm{mmW})$ technologies is a possible solution. In fact, despite the higher path-loss brought by the technological shift towards higher frequencies (e.g. at $60 \mathrm{GHz}$ ), the requirements in terms of power emission are less stringent [8] than those in the UWB band [9] with an available bandwidth up to $7 \mathrm{GHz}$ [1], [10]. Currently, only few preliminary studies concern the joint adoption of such technologies, which are at their infancy [11]-[13]. In particular, [12] showed a tag which harvests energy from the $\mathrm{mmW}$ signal transmitted by the reader, and successively exploits a $60 \mathrm{GHz}$ free-running oscillator to transmit back pulse-width modulated bursts. In [13] instead, the use of a standard UHF-RFID architecture, compatible with that of mmW-RFID, has been proposed. Unfortunately, due to the proposed schemes, the achievable reader-tag ranges are strongly limited by the path-loss.

We aim here to study the feasibility and the opportunities that can be offered by the joint adoption of $\mathrm{mmW}$ and RFID technologies when ad-hoc reader-tag architectures are exploited [14], and tags are assumed to be woken-up from stand-by mode by exploiting a dedicated signal as detailed in [5]. In particular, we first describe the considered scheme, and successively we present a measurement campaign conducted at $60 \mathrm{GHz}$ in order to enable a preliminary validation of the proposed system through both simulated and measured data.

Note that if near-pencil beam mmW antennas are exploited, the considered scheme could enable tags localization with only one reader which performs beamsteering, with the multi-tags 


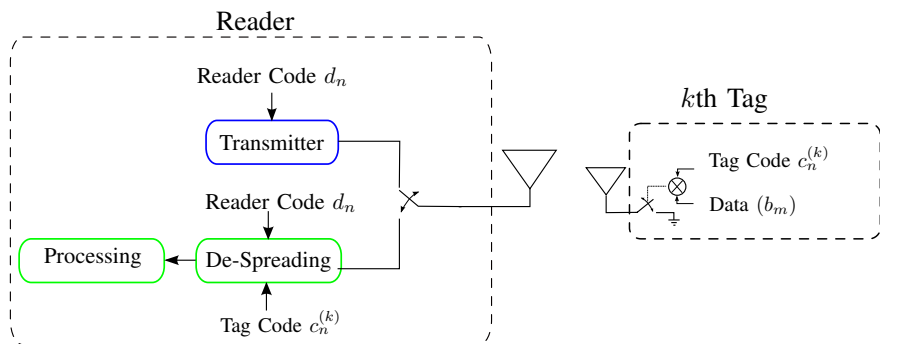

Fig. 2. Reader-Tag considered architectures for detection and communication.

interference phenomenon [14] drastically reduced [15].

The rest of the paper is organized as follows. In Sec. II, the considered reader-tag signalling scheme and the readertag architectures here exploited are described; successively the measurement campaign is described in III. Finally, numerical results are reported in Sec. IV and conclusions and future perspectives end the paper.

\section{SySTEM ARCHITECTURE}

\section{A. Backscattering Principle}

The reader-tag communication herein considered is based on the modulation of the backscattered signal. Usually, tags are in sleeping mode and they are woken-up thanks to the transmission of a wake-up signal (e.g., an unmodulated UHF carrier [5]). Once the wake-up procedure is completed, the reader transmits an interrogation pulse train, which is reflected back by tags and objects (see Fig. 1 and Fig. 2). In particular, the signals backscattered by tags are composed of two parts: the structural and the antenna mode component, as depicted in Fig. 1 [6]. While the former depends only on the antenna and support shape and materials, the latter is strictly related to how the antenna is loaded and thus it is the part of interest. In the ideal case, the antenna mode scattered waveforms present a phase difference of $180^{\circ}$ between the case of open and short circuit loads, which are usually adopted to discriminate different data symbols. Unfortunately, such signals are usually superimposed with those signals scattered by the surrounding environment (clutter), which could be even a few order of magnitude higher. To counteract such effects, ad-hoc signal processing algorithms have to be conceived in order to achieve long reader-tag reading distances.

\section{B. Transmitted Signal Model}

The reader is composed of a transmitter and a receiver section both connected to the same antenna through a TX/RX switch. According to the number of symbols $N_{\mathrm{b}}$ in the data packet, during the interrogation phase the reader transmits a sequence of $N_{\mathrm{b}} \cdot N_{\mathrm{s}}$ pulses, each having energy $E_{\mathrm{p}}$, modulated by the specific reader code, in the form

$$
s(t)=\sum_{m=0}^{N_{\mathrm{b}}-1} \sum_{n=0}^{N_{\mathrm{s}}-1} d_{n} p\left(t-n T_{\mathrm{p}}-m T_{\mathrm{s}}\right)
$$

where $\left\{d_{n}\right\}$ is the spreading code of the reader with length $N_{\mathrm{s}}$, and $T_{\mathrm{s}}, T_{\mathrm{p}}$ are the symbol and frame time, respectively. ${ }^{1}$

The signal received by the $k$ th tag is then modulated according to a bipolar code $\left\{c_{n}^{(k)}\right\}$, for $n=0,1, \ldots, N_{\mathrm{s}}-1$ and to the data $\left\{b_{m}\right\}$ stored inside it. To extremely simplify the architecture, the backscatter modulator reduces to a simple switch (see Fig. 2). Operating in such a manner it is possible to discriminate a specific reader-tag couple among all the possible combinations. Moreover, the choice of a zero mean code (that is, with the same number of +1 and -1 ) allows the clutter suppression if the received signals composing one symbol are coherently accumulated [14].

In addition, note that the longer the code sequence $N_{\mathrm{s}}$, the higher the reading range because of the higher accumulated signal-to-noise ratio (SNR). On the other side, $N_{\mathrm{S}}$ should be limited mainly for two reasons: to reduce the power consumption (i.e., the tag active time), and to reduce the effect of clock drift during the SNR accumulation.

In the following, we describe two possible architectures to post-process the received signal.

\section{Receiver Architecture}

We foresee the adoption of two possible classical receivers for mmW-RFID: a matched filter (MF), exploitable for $k$ th tag data demodulation, and an energy detector (ED), applicable in all those scenarios requiring low complexity.

1) Matched-Filter Receiver: Define $w(t)$ as the antenna mode backscattered response to the transmitted pulse $p(t)$, which is modulated by the load, and $w^{(\mathrm{c})}(t)$ as the clutter response. When the previously defined scheme is adopted, the $k$ th tag signal of interest results modulated by both the tag code and the information symbol. The received signal can thus be written as

$$
\begin{aligned}
r(t)= & \sum_{k \in \mathcal{T}} \sum_{m=0}^{N_{\mathrm{b}}-1} \sum_{n=0}^{N_{\mathrm{s}}-1} d_{n} c_{n}^{(k)} b_{m} w^{(k)}\left(t-n T_{\mathrm{p}}-m T_{\mathrm{s}}\right) \\
& +\sum_{m=0}^{N_{\mathrm{b}}-1} \sum_{n=0}^{N_{\mathrm{s}}-1} d_{n} w^{(\mathrm{c})}\left(t-n T_{\mathrm{p}}-m T_{\mathrm{s}}\right)+n(t)
\end{aligned}
$$

with $\mathcal{T}$ being the set of the tags in the environment.

The received signal is first passed through a filter having impulse response $h^{(\mathrm{MF})}(t)$ to obtain $v(t)=r(t) \otimes h^{(\mathrm{MF})}(t)$, where $\otimes$ is the convolution operator and $h^{(\mathrm{MF})}(t)$ is a local replica template of the received antenna mode component. ${ }^{2}$ Then, by assuming the intended tag is present, and by supposing perfect code and pulse synchronization, the output of the MF is sampled at time intervals $t_{l, m}=l T_{\mathrm{p}}+m T_{\mathrm{s}}+\tau_{0},{ }^{3}$ with $l=0, \ldots, N_{\mathrm{s}}-1$, thus obtaining the samples $v_{l, m}$ [4]. In order to remove the clutter contribution and the interference from other tags, the sampled values are multiplied by the

\footnotetext{
${ }^{1}$ According to [14], the analysis can be extended to multiple readers.

${ }^{2}$ In practical applications, it could be more favorable to down-convert the received signal with a mixer and perform base-band post-processing to reduce the receiver complexity.

${ }^{3}$ We assume that a perfect time-of-arrival (TOA) estimate is available, and consequently the time instant $\tau_{0}$ is perfectly chosen.
} 
reader and $k$ th useful tag composite sequence and successively accumulated. Thus we can write for the $m$ th symbol

$$
y_{m}=\sum_{l=0}^{N_{\mathrm{s}}-1} c_{l}^{(k)} d_{l} v_{l, m}=b_{m} N_{\mathrm{s}} E_{\mathrm{r}}+z_{m}
$$

where $E_{\mathrm{r}}$ is the collected energy per pulse from the $k$ th tag according to the considered template, the clutter has been suppressed thanks to the considered zero-mean codes which give $\sum_{l=0}^{N_{\mathrm{s}}-1} c_{l}^{(k)}=0$ and $z_{m}$ is the thermal noise at the output of the processing scheme. ${ }^{4}$ Among the possible choices for $h^{(\mathrm{MF})}(t)$, the simplest solution is the single-path matched filter where the template is chosen to be proportional to the received antenna mode backscattered component at a reference distance and in free-space propagation. In that case, $E_{\mathrm{r}}$ reduces with good approximation to the energy of the first received path in line-of-sight conditions [4]. ${ }^{5}$

According to [4], for the considered scheme the bit error probability (BEP) can be written as

$$
P_{\mathrm{b}}=\frac{1}{2} \operatorname{erfc} \sqrt{\frac{E_{\mathrm{r}} N_{\mathrm{s}}}{N_{0}}}
$$

where $\operatorname{erfc}(\cdot)$ is the complementary error function and $N_{0}$ is the noise power spectral density. Consequently, the received energy per symbol in ideal conditions is $E_{\mathrm{s}}=N_{\mathrm{s}} E_{\mathrm{r}}$ and, analogously, (4) can be written as

$$
P_{\mathrm{b}}=\frac{1}{2} \operatorname{erfc} \sqrt{\frac{P_{\mathrm{r}}}{N_{0} R_{\mathrm{b}}}}
$$

where $R_{\mathrm{b}}=1 /\left(N_{\mathrm{s}} T_{\mathrm{p}}\right)$ is the data rate, and $P_{\mathrm{r}}$ accounts for the transmitted power and the round-trip channel power gain.

Finally, in order to find the required number of pulses to achieve a certain reader-tag distance for a target $P_{\mathrm{b}}^{\star}$, it is possible to write (4) as

$$
N_{\mathrm{s}} \geq \hat{N}_{\mathrm{s}}=\left\lceil\frac{N_{0} \cdot\left(\operatorname{erfc}^{-1}\left(2 P_{\mathrm{b}}^{\star}\right)\right)^{2}}{E_{\mathrm{r}}}\right\rceil
$$

which puts in relation the minimum required number of pulses per symbol $\hat{N}_{\mathrm{s}}$ with the received energy $E_{\mathrm{r}}$.

2) Energy Detector: A simple and practical solution for wideband RFID, especially when the overall system complexity has to be kept low, is represented by the ED architecture which exploits the presence of a square-law device. Since the task here is only to accomplish tag detection, the receiver performs the coherent accumulation of the received signals corresponding to the $N_{\mathrm{s}}$ transmitted pulses $p(t)$ within a symbol [14]. As for the previous scenario, we assume to have the clock of readers and tags synchronous, and the $k$ th tag as the intended useful one. Then, the signal received back by the reader is expressed as in (2), with $b_{m}=1 \forall m$, which can

\footnotetext{
${ }^{4}$ Several codes have been proposed for multi-tag interference suppression [14]. Here, since the system is assumed to be perfectly synchronous, classical orthogonal codes for readers and tags can be considered.

${ }^{5}$ It is important to notice that the performance of this solution, and of the others accounting for Rake fingers synchronized to different paths of the received signal, is bounded by ideal matched filters able to collect the entire received energy, that is $E_{\mathrm{r}}=\int_{T_{\mathrm{p}}} w^{2}(t) d t$.
}

thus be neglected. According to [14], first the received signal is passed through a band-pass filter to eliminate the out-ofband noise, obtaining $\tilde{r}(t)$. Once the signal is filtered, it is de-spread and accumulated, giving

$$
y(t)=\sum_{l=0}^{N_{\mathrm{s}}-1} c_{l}^{(k)} \tilde{r}\left(t+l T_{\mathrm{p}}\right)
$$

with $t \in\left[0, T_{\mathrm{p}}\right]$. From (7), by passing $y(t)$ through an ED, we obtain a vector of energy bins, where each element can be expressed by

$$
e_{b}=\int_{(b-1) T_{\mathrm{ED}}}^{b T_{\mathrm{ED}}}[y(t)]^{2} d t
$$

with $b=1,2, \ldots, N_{\text {bins }}, T_{\mathrm{ED}}$ being the integration time, and $N_{\text {bins }}=\left\lfloor T_{\mathrm{p}} / T_{\mathrm{ED}}\right\rfloor$ is the number of bins at the output of the ED. Successively, each element $e_{b}$ is compared with a threshold. If there is at least one element overcoming the threshold, then the tag is assumed detected. Consequently, we have two pieces of information: the $k$ th tag received energy, which can be obtained by the bin value, and the TOA of the received signal, given by the bin index.

There are two figures of merit which can be adopted to evaluate the performance of the considered detection approach: the probability of false alarm (PFA) and the probability of detection (PD). The former stems from the probability to wrongly detect a tag even if it is not present in the scenario, whereas the latter to correctly detect its presence when effectively the intended useful tag is in the considered scenario. To this purpose, performance of passive UWB-RFID has been extensively characterized in terms of PFA and PD [14]. In particular, it has been shown that it is possible to achieve the desired performance in terms of PFA and PD by setting the minimum required number of pulses per symbol as

$$
N_{\mathrm{s}} \geq \hat{N}_{\mathrm{s}}=\left\lceil\frac{N_{0}}{2 E_{\mathrm{r}}}\left[Q_{h}^{-1}\left(P_{\mathrm{D}}^{\star}, \sqrt{\xi}\right)\right]^{2}\right\rceil
$$

where $Q_{h}$ is Marcum- $Q$ function of order $h$ [14], $P_{\mathrm{D}}^{\star}$ is the apriori desired PD and $\xi$ is the threshold set for a-priori desired $\operatorname{PFA}\left(P_{\mathrm{FA}}^{\star}\right)^{6}$

\section{III. $60 \mathrm{GHz}$ MEASUREMENT CAMPAIGN}

In order to validate the considered RFID architecture at $\mathrm{mmW}$, a measurements campaign has been conducted at CEAGrenoble indoor premises (see Fig. 3 and 4.). In particular, we measured the $S_{21}(f)$ parameter by placing the reader antenna at different distances from the tag antenna, with the reader antenna pointing towards the tag, as reported in Fig. 4. From this measure, the two-way channel comprising the reader and tag antennas gain can be easily found as $H(f)=S_{21}^{2}(f)$ [16]. Note that the one-way channel is obviously easier to measure than a two-way backscattering channel in terms of SNR. This channel transfer function accounts for the antenna mode

\footnotetext{
${ }^{6}$ For the herein considered case, $\xi$ does not account for interference signal
} and for any system non-ideality, but only for the receiver noise. 


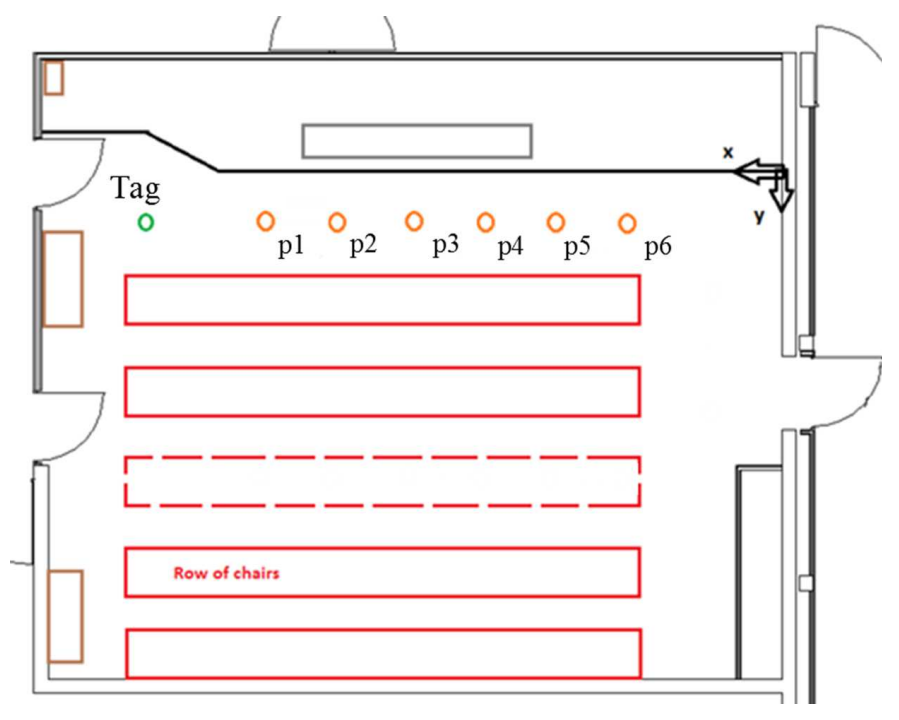

Fig. 3. Picture with the reader and tag antennas positions.

TABLE I

CONSIDERED MEASUREMENT PARAMETERS.

\begin{tabular}{|c|c|}
\hline Parameter & Value \\
\hline Center Frequency [GHz] & $f_{\mathrm{c}}=62$ \\
Bandwidth $[\mathrm{GHz}]$ & $\mathrm{BW}=6$ \\
Frequency points & 2001 \\
$d_{\mathrm{tag}-p_{1}}[\mathrm{~m}]$ & 2.7 \\
$d_{\mathrm{tag}-p_{2}}[\mathrm{~m}]$ & 3.5 \\
$d_{\mathrm{tag}-p_{3}}[\mathrm{~m}]$ & 4.3 \\
$d_{\mathrm{tag}-p_{4}}[\mathrm{~m}]$ & 5.1 \\
$d_{\mathrm{tag}-p_{5}}[\mathrm{~m}]$ & 5.9 \\
$d_{\mathrm{tag}-p_{6}}[\mathrm{~m}]$ & 6.7 \\
\hline
\end{tabular}

component only, without the superposition of the structural mode component and of the clutter.

The exploited measurement set-up consists of a 4-ports Vector Network Analyzer (VNA) operating in the frequency range $10 \mathrm{MHz}-24 \mathrm{GHz}$ connected both to the reader antenna (a horn with $20 \mathrm{dBi}$ gain and half power beamwidth (HPBW) of $20^{\circ}$ ) and to the tag antenna (a $0 \mathrm{dBi}$ gain omni-antenna, reported in Fig. 4), respectively, thanks to two $\mathrm{mmW}$ converters operating in the frequency range $50 \mathrm{GHz}-75 \mathrm{GHz}$. Consequently, a central frequency $f_{\mathrm{c}}=62 \mathrm{GHz}$ and bandwidth of $6 \mathrm{GHz}$ have been considered.

Table I presents the reader-tag distances considered in the conference room scenario with respect to the tag position, as reported in Fig. 3, which vary from $2.7 \mathrm{~m}$ to $6.7 \mathrm{~m}$ with a step of $0.8 \mathrm{~m}$. For the planned set of measurements, the reader orientation has been kept constant towards the tag. Moreover, for each measurement point, co-polar and crosspolar measurements have been carried out. The cross-polar data have been obtained by rotating the reader horn antenna by $90^{\circ}$ with the help of a waveguide twist. In this way, we emulated the presence of a tag attached to an object which has been rotated from its expected working condition. In such a way, performance can result to be drastically degraded as shown in the next section.

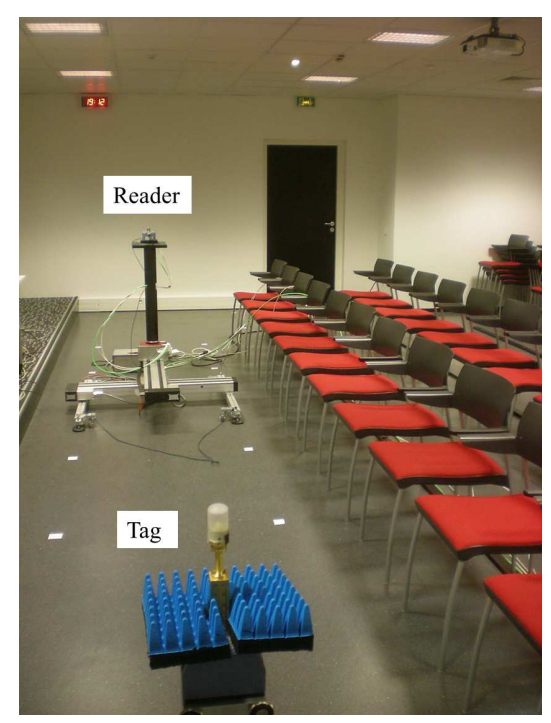

Fig. 4. Measurement scenario.

TABLE II

SIMULATION PARAMETERS.

\begin{tabular}{|c|c|c|}
\hline Parameter & UWB Value & $\mathrm{mmW}$ Value \\
\hline EIRP & $-11 \mathrm{dBm}$ & $30 \mathrm{dBm}$ \\
Bandwidth $W$ & $2 \mathrm{GHz}$ & $2 \mathrm{GHz}$ \\
Center frequency $f_{\mathrm{c}}$ & $4 \mathrm{GHz}$ & $60 \mathrm{GHz}$ \\
Time frame $T_{\mathrm{p}}$ & $128 \mathrm{~ns}$ & $128 \mathrm{~ns}$ \\
Reader antenna gain $G_{\mathrm{r}}$ & $5 \mathrm{dBi}$ & $20 \mathrm{dBi}$ \\
Tag antenna gain $G_{\mathrm{t}}$ & $0 \mathrm{dBi}$ & $0 \mathrm{dBi}$ \\
Tag losses $L_{\mathrm{t}}$ & $2 \mathrm{~dB}$ & $2 \mathrm{~dB}$ \\
Receiver noise figure $F$ & $4 \mathrm{~dB}$ & $4 \mathrm{~dB}$ \\
Desired PFA $P_{\mathrm{FA}}^{\star}$ & $10^{-3}$ & $10^{-3}$ \\
Desired PD $P_{\mathrm{D}}^{\star}$ & 0.9 & 0.9 \\
Desired BEP $P_{\mathrm{b}}^{\star}$ & $10^{-3}$ & $10^{-3}$ \\
\hline
\end{tabular}

\section{RESUlts}

In this section we characterize the performance of the passive mmW-RFID through both simulation and measurement data. In particular, the comparison with the UWB backscattering based technology is performed in order to assess preliminary system performance. Results are obtained by means of (5), (6) and (9), for a target $P_{\mathrm{b}}^{\star}=10^{-3}$, a $P_{\mathrm{FA}}^{\star}=10^{-3}$ and a $P_{\mathrm{D}}^{\star}=0.9$.

\section{A. Simulation Results}

We first compare through simulations the performance of UWB and mmW-RFID in the additive white Gaussian noise (AWGN) scenario. In both cases, we considered the parameters reported in Table II, and a transmitted pulse centered at $4 \mathrm{GHz}$ and $60 \mathrm{GHz}$, respectively, compliant with the Federal Communications Commission (FCC) mask in the two different bands [8], [9]. The system parameters were selected according to [6] for UWB. Consequently, by moving towards $60 \mathrm{GHz}$, we experience the variation of two parameters: the effective radiated isotropic power (EIRP), which is less stringent at $60 \mathrm{GHz}$ with respect to lower frequencies [8], and the reader antenna gain which is higher due to the adopted antennas. For 


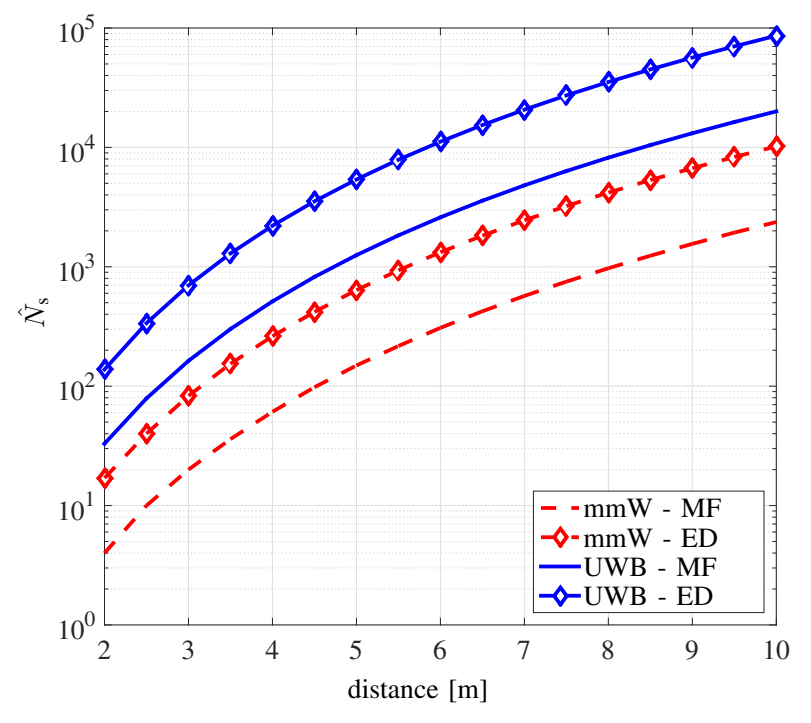

Fig. 5. Required number of pulses for different receiver architectures.

what the ED is concerned, we considered an ideal case where all the received energy is contained in one bin.

Figure 5 shows the values of $\hat{N}_{\mathrm{S}}$ for the two receiver architectures. Note that under the EU mask constraint for UWB, a reading range of about $6.5 \mathrm{~m}$ can be achieved adopting $\hat{N}_{\mathrm{s}} \simeq 1.5 \cdot 10^{4}$ for an ED, whereas only around $2 \cdot 10^{3}$ pulses are required for the same distance and conditions at $\mathrm{mmW}$. We have different competing effects: if from one side, $\mathrm{mmW}$ RFID suffers from the high path-loss experienced at $60 \mathrm{GHz}$, from the other side it benefits from the less stringent power regulations than at $4 \mathrm{GHz}$ [9]. In this way, if one of the main requirements is the system refresh rate, which is given by $R_{\mathrm{f}}=1 /\left(T_{\mathrm{s}} N_{\mathrm{b}}\right)$, mmW-RFID is more promising than a UWB solution as $T_{\mathrm{S}}$ can be reduced for a given reader-tag distance. Such advantage is mainly given by the significantly power emission allowed in the $\mathrm{mmW}$ band to which more energy consumption corresponds.

Fig. 6 shows the obtained BEP as a function of the different data rates for passive mmW-RFID by using (5). If we fix the target $\mathrm{BEP}$ to $10^{-3}$, results show that data rates in the order of $2 \mathrm{Mbit} / \mathrm{s}$ for distances around $2 \mathrm{~m}$ are achievable.

\section{B. Measurement Results}

We here exploit measured data to assess the feasibility of the proposed reader-tag communication scheme. In particular, the backscattering channel impulse response has been convoluted with a root raised cosine pulse centered at $60 \mathrm{GHz}$ and with bandwidth $\mathrm{BW}=2 \mathrm{GHz}$, compliant with the FCC mask. For the ED, we considered an ideal case scenario where the bin with $T_{\mathrm{ED}}=1 \mathrm{~ns}$ is centered to the received signal with the highest peak. ${ }^{7}$ Moreover, for the MF we consider a single path MF whose template is perfectly matched to the received maximum peak pulse, without accounting for pulse distortion

\footnotetext{
${ }^{7}$ With good approximation, it can be associated to the first path for the considered scenario.
}

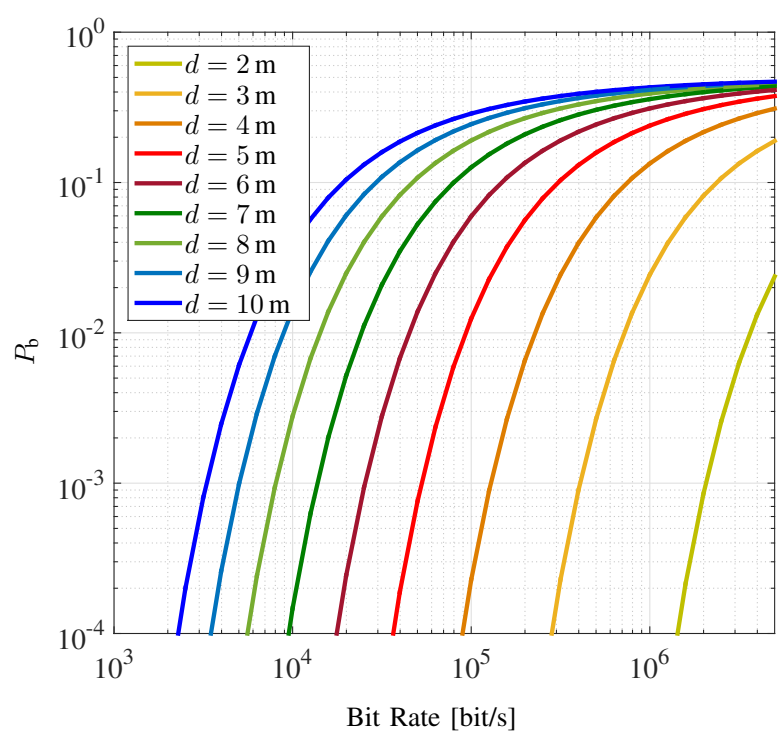

Fig. 6. Obtained data rates for different reader-tag distances.

[4]. In addition, we compare the performance for co-polar and cross-polar measurements, thus simulating the two opposite scenarios for a tag randomly attached to an object.

Fig. 7 reports $\hat{N}_{\mathrm{s}}$ for the two receiver architectures. Now, with $\hat{N}_{\mathrm{s}} \simeq 10^{3}$, it is possible to achieve $p_{6}=6.7 \mathrm{~m}$ for a MF, whereas around $10^{4}$ pulses are required for tag detection with an ED architecture. Thus, the performance is a bit degraded with respect to the AWGN scenario because of the simplifying assumptions done in simulations, that is the entire received energy is contained in one bin. On the contrary, it is expected that performance can be improved if architectures closer to ideal matched filters are exploited, thus giving the opportunity to collect the received energy from different multipath components. Note that cross-polar configurations need a really high number of accumulated pulses. This effect is detrimental when the tag orientation is not known or could change. In controlled environments (that is, where the tag orientation is always kept the same) this result could be exploited to limit the interference of close different reader-tag devices to cope with the high polarization loss.

As far as the localization capability is concerned, the adoption of near-pencil beam antennas at reader side could enable the opportunity to perform tags localization through beamsteering operations as well as to further increase the link budget and reduce the clutter. In this way, reliable localization performance is expected to be guaranteed by exploiting a single reader beamsteering [17], and future scenarios can foresee the integration of such mmW-RFID readers into portable devices to search and localize the surrounding tags [15].

\section{CONCLUSION}

In this paper we investigated the possibility to adopt $\mathrm{mmW}$ technology for passive RFID systems. After briefing introducing the proposed architectures and a $\mathrm{mmW}$ measurement campaign, we evaluated the attainable performance by comparing 


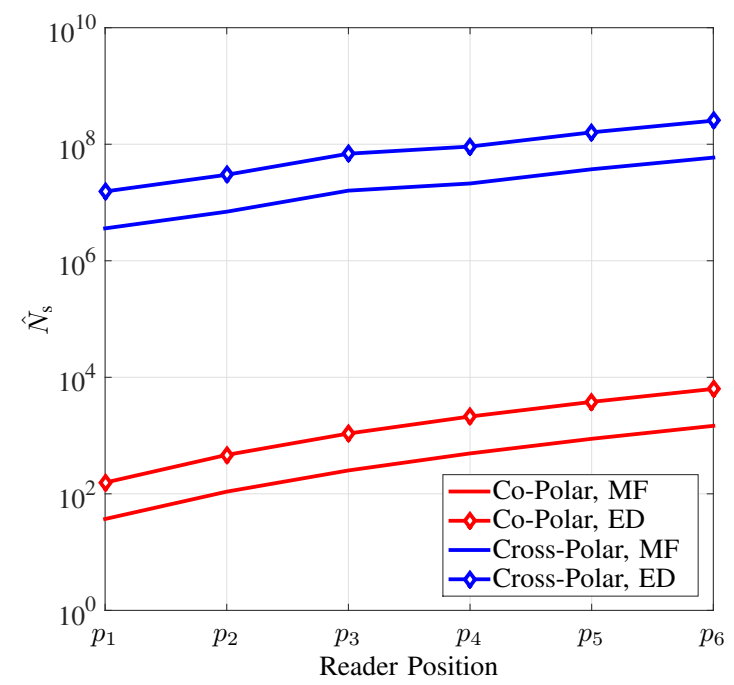

Fig. 7. Required number of pulses for different receiver architectures using measured data at $60 \mathrm{GHz}$.

results with those of UWB in the microwave band. Thanks to measured data, a preliminary system characterization has been also performed in a real environment. In ideal conditions we showed that the proposed scheme can outperform the UWB one, because of requiring a lower number of pulses to achieve the same reader-tag distance and consequently a reduced complexity.

Future works are expected to investigate ad-hoc tags wakeup procedures, $\mathrm{mmW}$ energy-harvesting possibilities, and adhoc low-complexity single reader localization schemes, as well as to extensively characterize the performance for different tags orientations, positions and measurement scenarios.

\section{ACKNOWLEDGMENTS}

This research was supported in part by the H2020 IFEF Marie-Curie project MAPS (Grant 659067) and by the European H2020 project XCycle (Grant 635975). Authors would like to thank Anna Guerra and Antonio Clemente for the fruitful discussions.

\section{REFERENCES}

[1] F. Boccardi et al., "Five disruptive technology directions for 5G," IEEE Commun. Mag., vol. 52, no. 2, pp. 74-80, Feb. 2014.

[2] Z. Zhang et al., "Item-level indoor localization with passive UHF RFID based on tag interaction analysis," IEEE Trans. Industrial Electron., vol. 61 , no. 4, pp. 2122-2135, Apr. 2014.

[3] D. Arnitz, U. Muehlmann, and K Witrisal, "Characterization and modeling of UHF RFID channels for ranging and localization," IEEE Trans. Antennas Propag., vol. 60, no. 5, pp. 2491-2501, May 2012.

[4] D. Dardari et al., "Ultrawide bandwidth RFID: The next generation?" Proc. IEEE, vol. 98, no. 9, pp. 1570-1582, Sep. 2010.

[5] R. D'Errico et al., "An UWB-UHF semi-passive RFID system for localization and tracking applications," in Proc. IEEE Int. Conf. on RFIDTechnology and Applications (RFID-TA), Nice, France, Nov. 2012, pp. $1-6$.

[6] N. Decarli, F. Guidi, and D. Dardari, "Passive UWB RFID for tag localization: Architectures and design," IEEE Sensors J., vol. 16, no. 5, pp. 1385-1397, Mar. 2016.
[7] F. Guidi et al., "Analysis of UWB tag backscattering and its impact on the detection coverage," IEEE Trans. Antennas Propag., vol. 62, no. 8, pp. 4292-4303, Aug. 2014.

[8] "Revision of Part 15 of the Commissions Rules Regarding Operation in the 57-64 GHz Band," Federal Communications Commission (FCC), Aug. 2013.

[9] FCC, "Revision of Part 15 of the Commission Rules Regarding UltraWideband Transmission Systems, First Report and Order, ET Docket 98-153, FCC 02-8, adopted/released Feb. 14/Apr. 22, 2002 ".

[10] T. Rappaport et al., "Millimeter wave wireless communications." Prentice Hall, 2014.

[11] P. Pursula et al., "Millimeter-wave identification - a new short-range radio system for low-power high data-rate applications," IEEE Trans. Microw. Theory Tech., vol. 56, no. 10, pp. 2221-2228, Oct. 2008.

[12] S. Pellerano, J. Alvarado Jr, and Y. Palaskas, "A mm-wave powerharvesting RFID tag in $90 \mathrm{~nm}$ CMOS," IEEE J. Solid-State Circuits, vol. 45, no. 8, pp. 1627-1637, Aug. 2010.

[13] P. Pursula, F. Donzelli, and H. Seppa, "Passive RFID at millimeter waves," IEEE Trans. Microw. Theory Tech., vol. 59, no. 8, pp. 21512157, Aug. 2011.

[14] F. Guidi et al., "Detection of multiple tags based on impulsive backscattered signals," IEEE Trans. Commun., vol. 62, no. 11, pp. 3918-3930, Nov. 2014.

[15] D. Dardari et al., "The future of Ultra-Wideband localization in RFID," in Proc. IEEE Int. Conf. on RFID (IEEE RFID), Orlando, USA, May 2016.

[16] R. D'Errico, “An indoor backscattering channel characterization for UWB passive RFID applications," in Proc. 6th European Conf. on Antennas and Propag. (EUCAP), Mar. 2012, pp. 1169-1173.

[17] A. Guerra, F. Guidi, and D. Dardari, "Position and orientation error bound for wideband massive antenna arrays," in Proc. IEEE Int. Conf on Commun. (ICC), ANLN Workshop, 2015 